\title{
Fluid-induced Vibrations Test of Hydraulic Plane Gate for Different Conditions
}

\author{
Tingting Yang ${ }^{\mathrm{a}}$, Shihua $\mathrm{He}^{\mathrm{b}}$, Chunying Shen ${ }^{\mathrm{c}}$ and Qi Yang ${ }^{\mathrm{d}}$ \\ Faculty of Electric Power Engineering, Kunming univeisity of science and technology, Kunming, \\ 650500, China \\ a912832062@qq.com, bhoxiwa@aliyun.com, '86295700@qq.com, ${ }^{\mathrm{a}} 545715081 @ q q . c o m$
}

\begin{abstract}
Key words: plane gate; fluid-induced vibration; PIV; flowing measurement
Abstract: The hydraulic test model was built to investigate the fluid-induced vibration of plane gate by changing the gate opening and flow rate respectively. The flow field near the gate was measured with the PIV system while the gate structure was measured with 8 channels high speed vibration acquisition system. In the experiments, the dynamic distribution characteristics of the flow field around gate and dynamics parameters of the gate structure were measured. The results show that the gate opening and flow rate affect the flow-induced vibration of gate directly. Gate vibration shows asymmetric and aperiodic coupling vibration characteristics. When the flow field interacts with gate, the flow field near the gate is further disturbed. The changes of the corresponding flow boundary conditions result in the downstream fluid showing complex turbulent motion.
\end{abstract}

\section{Introduction}

The hydraulic gate vibration often occurs in its opening and closing process or partial open. The reasons of gate vibration lie in two aspects. One is the effect of fluid force. Secondly, the structural rigidity of the gate is not enough [1].With the FEM and computer technology advances, numerical simulations can calculate the natural frequency of gate structure more accurately and predict the gate vibration characteristics. Literature [2-6] used numerical analysis method to analyze the impact of gate opening and hydraulic head on natural frequencies and vibration modes by establishing finite element model. Jiao [7] who adopted test and finite element method to analyze the natural frequencies and mode shapes of the gate in different conditions discussed the effects of the added mass on the vibration characteristics. Thang [8] thought that gate vibration was caused by the downstream vortex action. Jongeling [9] put forward different interpretations that downstream secondary vortex didn't transport energy to gate and structure vibration was caused by the change of pressure formed by the unstable free shear layer of bottom edge. Dong's study [10] showed that gate vibration was mainly caused by the large-scale surface vortex impinging gate repeatedly. Hardwick [11] thought that shear layer destruction and unstable reattachment action of bottom edge produced the self-excited vibration under certain conditions.

Due to the complexity of flow-induced vibration and the limitations of the numerical analysis model, it is necessary to apply experiment method to reveal the internal mechanism of flow-induced vibration of the gate. The Particle Image Velocimetry (PIV) breaks through the limitation of a single-point test technology and can be used to measure the transient details of unsteady flow by comparing and analyzing the continuous multiple instantaneous velocities. PIV technology was widely used in experimental research in a variety of complex flow field [12 14]. However, the PIV is mainly restricted to a single fluid field testing which is difficult to grasp the mechanical behavior of fluid-structure coupling system. In this paper, the synchronous measurements both flow field and gate vibrating parameters are conducted by using the PIV system and DEWE-43A vibrating data acquisition system. The results can provide a hydrodynamic basis for hydraulic gate vibration problems and a reference for design and operation of gate. 


\section{Test set-up}

As shown in Figure 1, the experimental model system consists of the motor-pump system, flume and gate made of PMMA materials, and water supply tank. The entire apparatus is formed from a circulatory regulation system. The flow velocity is measured by PIV system which is a full three-dimensional particle velocimetry and consists of light source system, image acquisition system, control coordinate system, tracer particle generation system and display system. The vibration parameters of gate structure are measured by the DEWE-43A multi-channel data acquisition system which can perform accurate and reliable dynamic testing and analysis for displacement, velocity, acceleration and other physical variables.

The experiment is carried out in a plexiglass flume of $300 \mathrm{~cm}$ length, $10 \mathrm{~cm}$ width and $19 \mathrm{~cm}$ height. There is a right triangle weir in the first part of the flume which is used to measure the flow rate of water tank. Between the inlet and the static pond is contracted section of plexiglass baffle. A $24 \mathrm{~cm}$ high, $6 \mathrm{~mm}$ thick plane gate away from the inlet $10.1 \mathrm{~cm}$ is placed in the flume. The origin of coordinates is away from the flume bottom $10 \mathrm{~cm}$ and on the flume right wall along the flow direction. The $x-, y$-, and z-axes are along the flow, vertical, and wide directions of flume respectively. The accelerometers 1 and 3 are arranged at the middle gate $(z=5 \mathrm{~cm})$ and are $1 \mathrm{~cm}$ and $6 \mathrm{~cm}$ away from the bottom edge of the gate respectively.

The five set test parameters including head on weir, flow rate, gate opening, upstream and downstream levels are shown in Table 1.

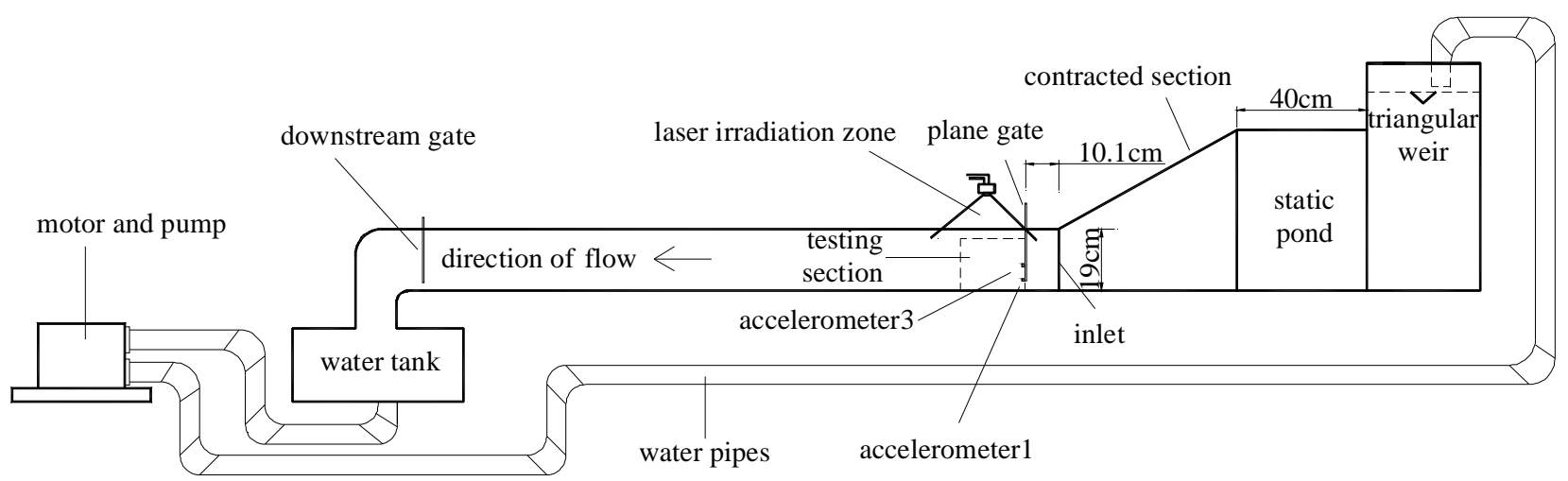

Fig.1 Schematic diagram of experimental set-up

Table 1Experimental parameters for different conditions

\begin{tabular}{cccccc}
\hline conditions & $\begin{array}{c}\text { head on weir } \\
{[\mathrm{cm}]}\end{array}$ & $\begin{array}{c}\text { flow rate } \\
{\left[\mathrm{cm}^{3} / \mathrm{s}\right]}\end{array}$ & $\begin{array}{c}\text { opening } \\
{[\mathrm{cm}]}\end{array}$ & $\begin{array}{c}\text { upstream } \\
\text { water level }[\mathrm{cm}]\end{array}$ & $\begin{array}{c}\text { downstream } \\
\text { water level }[\mathrm{cm}]\end{array}$ \\
\hline 1 & 7.8 & 2379 & 1.5 & 39.4 & 16.3 \\
2 & 7.8 & 2379 & 3 & 20.7 & 16.3 \\
3 & 7.8 & 2379 & 4.5 & 17.5 & 16.3 \\
4 & 9.3 & 3693 & 3 & 26.9 & 15.0 \\
5 & 6.8 & 1688 & 3 & 14.2 & 12.7 \\
\hline
\end{tabular}

\section{Test results and discussion}

Fig. 2 shows the displacement $d$ curves measured by acceleration sensors 1 and 3 for three different gate openings and a fixed water head $H=7.8 \mathrm{~cm}$. Fig. 3 and fig. 4 show the velocity $u$ and acceleration $a$ changed with time which are measured by acceleration sensor 1 . The following results can be seen from the figures. (1) The plane gate vibration is a non-symmetry and aperiodic 
state. (2) For different gate openings, the acceleration sensor 1 detects positive maximal displacement of $2.75 \times 10^{-4} \mathrm{~mm}$ and negative maximal displacement of $-2.64 \times 10^{-4} \mathrm{~mm}$. Acceleration sensor 3 detects positive maximum displacement of $2.61 \times 10^{-4} \mathrm{~mm}$ and negative maximum displacement of $-2.46 \times 10^{-4} \mathrm{~mm}$. All the maximal displacement appears at the smallest gate opening. When the water head is the same, the smaller the gate opening is, the larger the gate vibration amplitude is. (3) Throughout test period, the largest displacements of three different gate openings are captured at different moments and the largest displacements are 10 to 15 times higher than the average. Under the fluid-solid coupling vibration, resonance phenomenon occurs when water disturbance frequency and fundamental frequency of structure close to the same at a moment. (4) The gate vibration displacement peak appears in a particular moment. This phenomenon indicates that the vibrating structure in turn acts on the fluid and changes the flow characteristics of fluid, which leads the flow fluctuation frequency to deviate structural vibration frequency and changes the gate vibration displacement. (5) The changes of the velocity and acceleration of the gate also shows asymmetry and non-cyclical, which reflects the unsteady variation characteristics of flow-induced vibration.

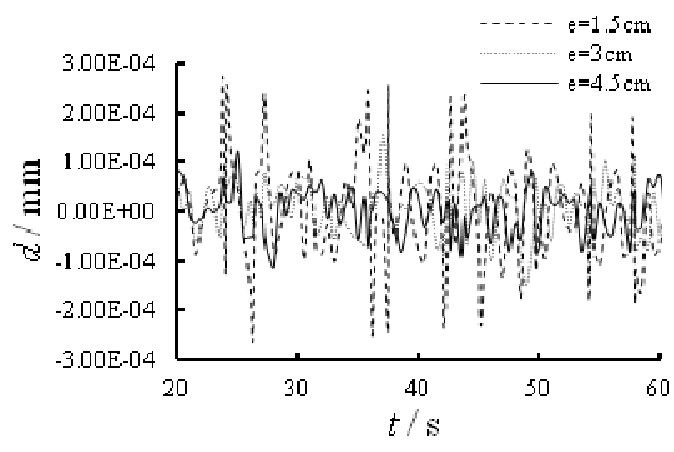

( a ) Vibration displacement curve of accelerometer 1

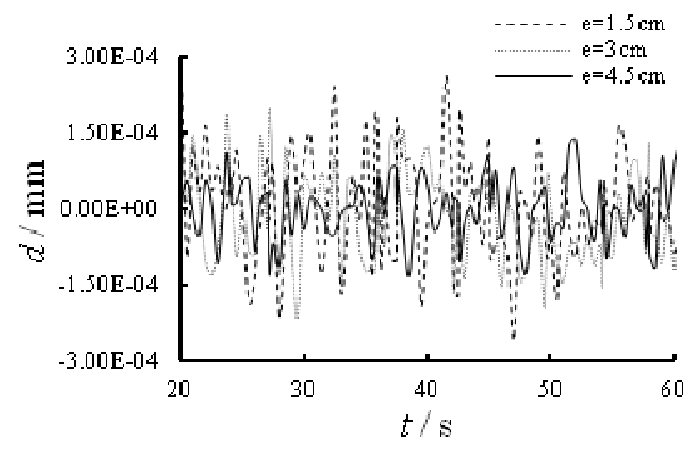

(b) Vibration displacement curve of accelerometer 3

Fig. 2 Vibrating displacement of gate for different openings

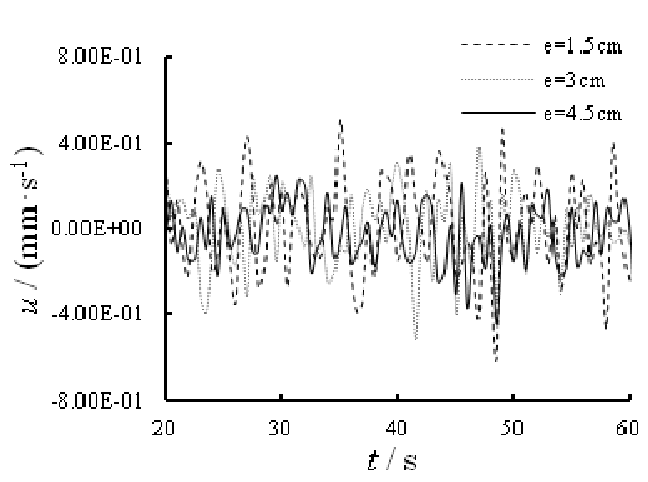

Fig. 3 Vibrating velocity of gate for different openings

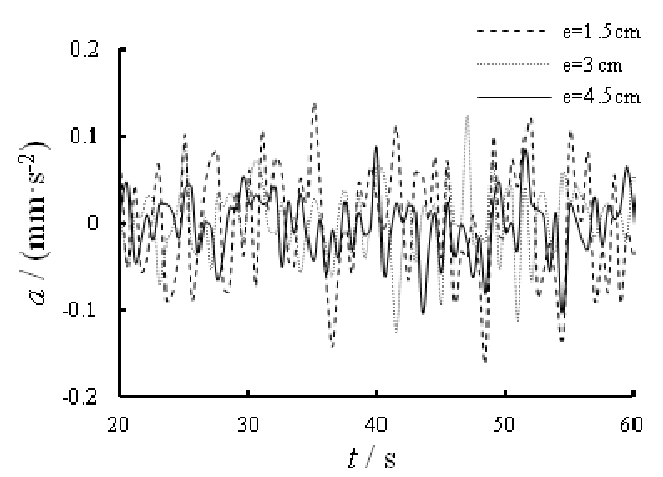

Fig. 4 Vibrating acceleration of gate for different openings

Fig. 5 shows the vibration displacement $d$ curve measured by accelerometer 1 and 3 for three different flow rates and unchanged gate opening $e=3 \mathrm{~cm}$. The following results can be seen from the figures 5 (a) and (b) . (1) The maximum amplitude appears when the flow rate is maximal, which indicates that the larger of the flow rate, the larger the maximal displacement of gate vibration for the same gate opening. (2) At the maximal flow rate, the acceleration sensor 1 measures the positive maximal displacement of $4.32 \times 10^{-4} \mathrm{~mm}$ and negative maximal displacement of $-3.56 \times 10^{-4} \mathrm{~mm}$. The acceleration sensor 3 measures 
positive maximal displacement of $2.26 \times 10^{-4} \mathrm{~mm}$ and negative maximal displacement of $-2.56 \times 10^{-4} \mathrm{~mm}$. Therefore, the action of fluid force on the bottom edge of gate is the strongest. (3) In the figure (a), vibration displacement at maximal flow rate is five times larger than the average of other two the flow rates, which indicates that higher water head, larger flow rate and smaller opening can cause stronger turbulent flow and greater vibration displacement of gate. (4) In the figure 5(b), the downstream level is the deepest when flow rate $Q=2379 \mathrm{~cm}^{3} / \mathrm{s}$ and the gate is reacted by the downstream surface turbulent flow where the accelerometer 3 located, resulting in the average amplitude of gate is greater within a certain time.

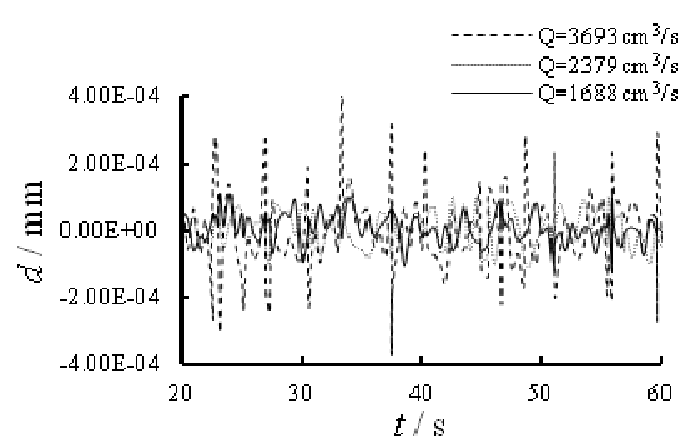

(a) Vibration displacement curve of accelerometer 1

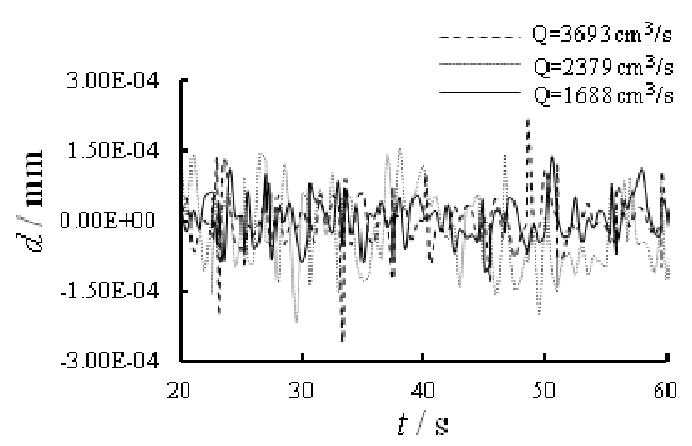

(b) Vibration displacement curve of accelerometer 3

Fig. 5 Vibrating displacement of gate for different flow rates

The curve of gate vibration displacement with time is shown in Figure 6 for the condition $Q=2379 \mathrm{~cm}^{3} / \mathrm{s}, e=1.5 \mathrm{~cm}$. Three special moments $t_{1}, t_{2}$, and $t_{3}$ are marked in the figure 6 which represent respectively the moments of equilibrium position, positive maximal displacement, negative maximal displacement of gate vibration. Figure 7 shows velocity vector profile corresponding to three specific moments. It can be seen that the magnitude and distribution of flow velocity after the gate are related with the vibration amplitude. Larger velocity and stronger tuebulent motion at the moments $t_{2}$ and $t_{3}$ lead to larger vibration displacement than that at the moments $t_{1}$.

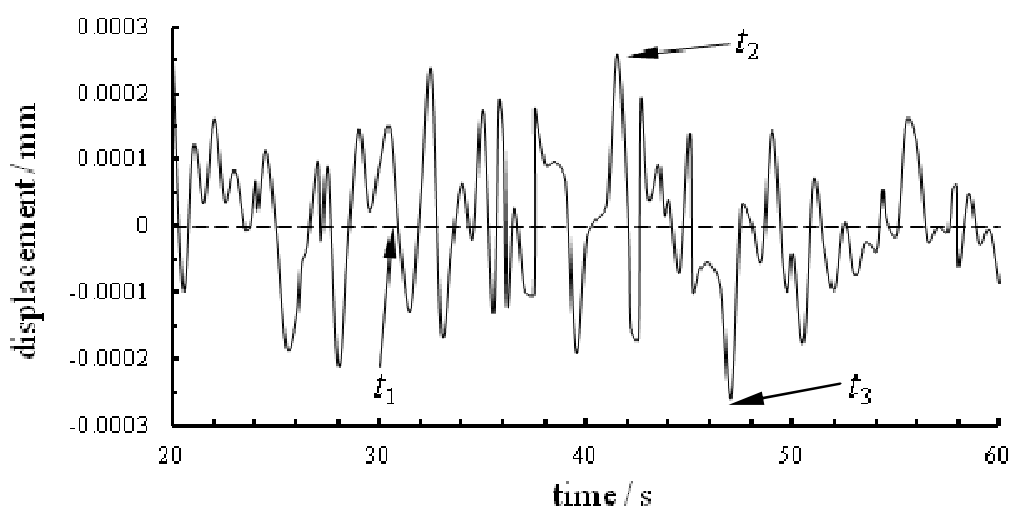

Fig.6 Vibrating displacement of gate

Figure 8 shows the velocity vector distribution of flow field for $Q=3693 \mathrm{~cm}^{3} / \mathrm{s}, e=3 \mathrm{~cm}$. Due to the instability of the flow itself and the effect of structural vibration on flow field, the flow quickly spreads through the gate hole and exchanges energy each other after combining with the downstream flow. The recirculation zone behind the gate and the different scale vortexes in almost parallel to the height position 
of the gate bottom edge can be observed, which leads to the different displacement of gate vibration at at different times and locations.

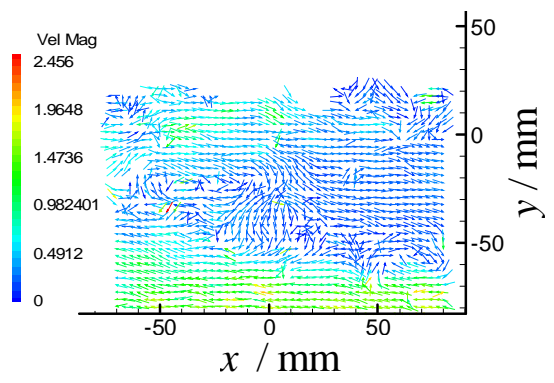

(a) $t_{1}=31.1 \mathrm{~s}$

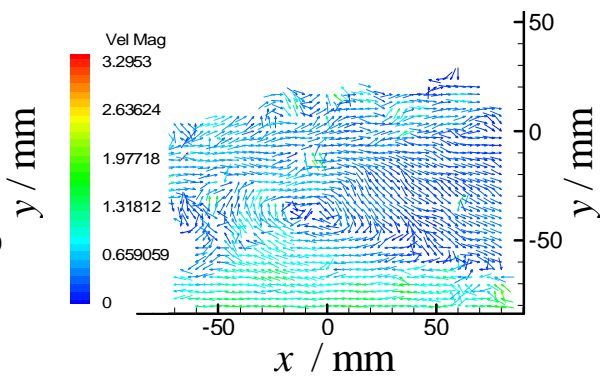

(b) $t_{2}=41.5 \mathrm{~s}$

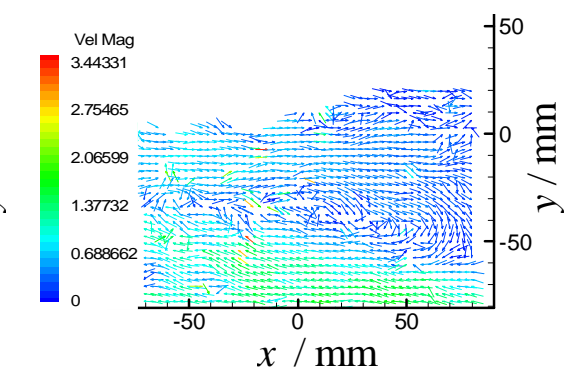

(c) $t_{3}=47.1 \mathrm{~s}$

Fig.7 Velocity field for $Q=2379 \mathrm{~cm}^{3} / \mathrm{s}, e=1.5 \mathrm{~cm}$

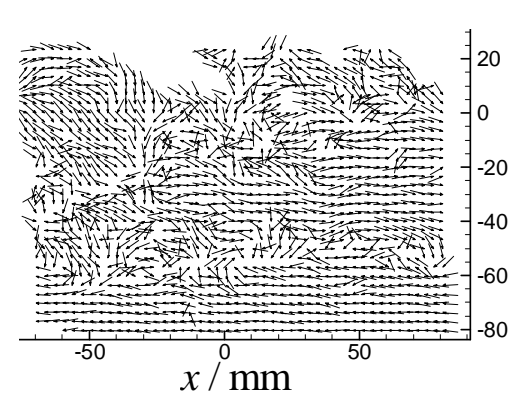

(a) $t=21.5 \mathrm{~s}$

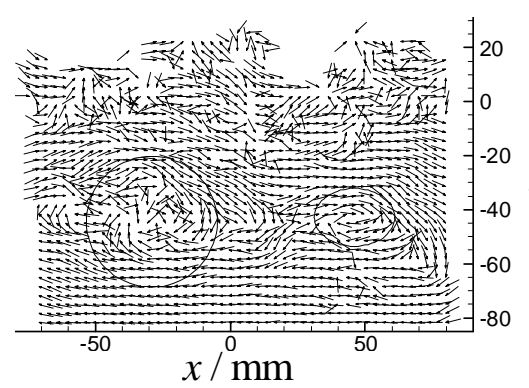

(d) $t=31.1 \mathrm{~s}$

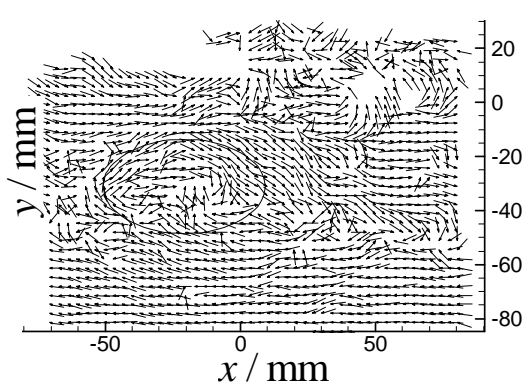

(b) $t=24.7 \mathrm{~s}$

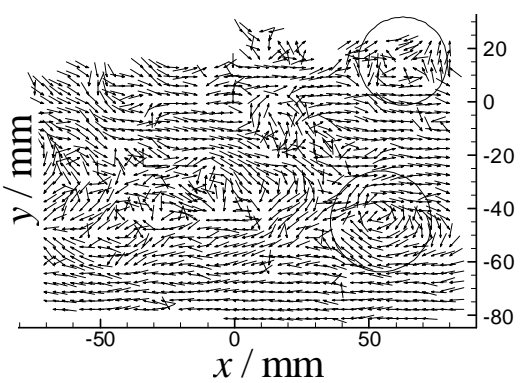

(e) $t=34.3 \mathrm{~s}$

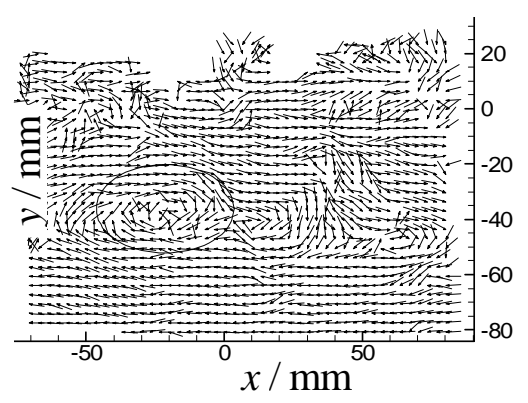

(c) $t=27.9 \mathrm{~s}$

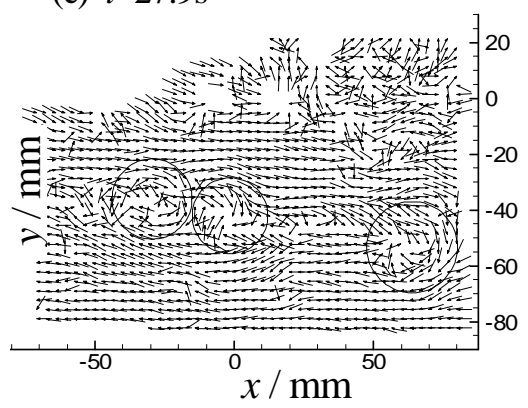

(f) $t=37.5 \mathrm{~s}$

Fig. 8 Velocity field for $Q=3693 \mathrm{~cm}^{3} / \mathrm{s}, e=3 \mathrm{~cm}$

\section{Conclusions}

For five different gate working conditions, the PIV and 8-channels high-speed dynamic vibration acquisition systems are applied to test plane gate vibration and flow field characteristics, the results are as follows.

(1) From the information of vibration test, the largest vibration displacement can be captured at a particular moment for different conditions. Flow-induced vibration of plane gate presents asymmetric and aperiodic characteristics. Gate opening and flow rate directly impact on the vibration displacement, velocity and acceleration. The smaller the gate opening is and the greater the flow rate is, the larger the gate vibration displacement is.

(2) The instantaneous velocity distributions of the flow field at a particular moment of gate vibration and the vortex dynamic evolution processes near the gate are obtained by PIV. The interaction effect 
between the flow field and the gate is captured. Gate vibration directly results in the different motion characteristics of the downstream flow field at different moments. Similarly, the variety of flow characteristics in turn changes the exciting force acting on the gate, which leads to varying vibration displacement at different moments.

(3) The turbulent flow movement at the height of the bottom edge of the gate is the strongest which leads to form various scale vortex structures at different moments. Vibration displacement on the bottom edge of the gate is the largest for different conditions.

\section{Acknowledgements}

This work was financially supported by the National Natural Science Foundation of China (51369013) and the Natural Science Foundation of Kunming University of Science and Technology (KKZ3201304016).

\section{References}

[1] J.J. Lian, X.M. Peng, G.T. CUI, et al. On the stability of gate vibrations [J]. Journal of Tianjin University, 32(2) (1999): 171-176.

[2] H.F. Xue, Y. Wang. Finite element analysis on natural dynamic of fluid-solid coupling vibration of plane gate based on ANSYS [J]. Journal of Anhui Agri. Sci., 40(8) (2012):5007-5009.

[3] Z.D. Xu, L.H. Du, J.M. Cai. Study on the free vibration characteristics of liquid-solid coupling of plane gate [J]. Hydraulic Electro generating, (4) (2001):39-43.

[4] H. Gu, G.H. Yan. Numerical analysis of natural vibration properties of hydraulic gate considering liquid-solid coupling [J]. Journal of Vibration, Measurement \&Diagnosis, 28(3) (2008): 242-301.

[5] Y.H. Wu, SZ. Xie. Dynamic characteristic analysis of interaction of fluid and hydraulic Structures [J]. Shui Li Xue Bao, (1) (1995): 27-34.

[6] S.J. Pan, X. Wang. Experimental study and numerical simulation on flow-induced vibration of large plate steel gate [J]. Water resources and power, 29(8) (2011):148-151.

[7] X.M. Jiao. Research on dynamic characteristic and flow-induced vibration of radial gate [D]. Wuhan: Wuhan University, 2005.

[8] N.D. Thang, Q.H. Lin, E. Naudasche. Flexural streamwise vibration of gate plates under vortex action [J]. Proc Int on Flow-Induced Vibrations, 37(2) (1987): 171-181.

[9] T.H.G. Jongeling. Flow-induced self-excited in-flow vibrations of gate plates [J]. Fluids and Structures, 2(1988): 541-566.

[10] S.F. Dong. Vibration mechanism of flate gate for submerged hydraulic jump [J]. Water Conservancy Science and Technology of Shan Dong, (1) (1998): 25-26.

[11] Hardwick. Flow-induced vibration of vertical-lift gate [J].Journal of the Hydraulics Division, 100(5) (1974):631-644.

[12] C.B. Jang, G. Wang, B. Deng, et al. PIV measurement of hydrodynamic characteristics of pier with a slot [J]. Advances in Water Science, 25(3) (2014):383-391.

[13] J. Wu, R. Qie, W. Li, et al. Research on vortexes of cross flow past a horizontal circular cylinder by PIV system [J]. Advances in Water Science, 16(5) (2005): 628-633. 
[14] H. Zhang, Z.R. Lu, S.D. Sun. The experimental study on unsteady horsedhoe vortex structure in juncture flow with PIV [J]. Chinese Journal of Theoretical and Applied Mechanics, 40(2) (2008): 171178. 